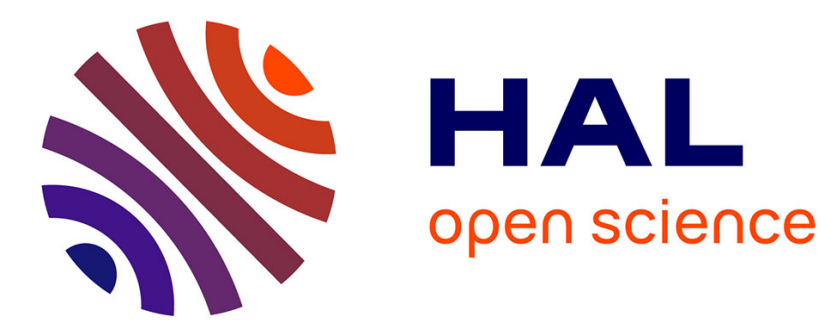

\title{
Digital control strategies for switch-mode power supply
}

Xuefang Lin-Shi, Bruno Allard, Dominique Tournier, Jean-Marie Rétif, Florent Morel

\section{To cite this version:}

Xuefang Lin-Shi, Bruno Allard, Dominique Tournier, Jean-Marie Rétif, Florent Morel. Digital control strategies for switch-mode power supply. 32nd IEEE IECON, Nov 2006, Paris, France. pp.79 - 84, 10.1109/IECON.2006.347547 . hal-00369390

\section{HAL Id: hal-00369390 \\ https://hal.science/hal-00369390}

Submitted on 3 Jun 2009

HAL is a multi-disciplinary open access archive for the deposit and dissemination of scientific research documents, whether they are published or not. The documents may come from teaching and research institutions in France or abroad, or from public or private research centers.
L'archive ouverte pluridisciplinaire HAL, est destinée au dépôt et à la diffusion de documents scientifiques de niveau recherche, publiés ou non, émanant des établissements d'enseignement et de recherche français ou étrangers, des laboratoires publics ou privés. 


\title{
Digital control strategies for switch-mode power supply
}

\author{
Xuefang Lin-Shi, Bruno Allard, Senior Member, IEEE, Dominique Tournier, \\ Jean-Marie Rétif, Florent Morel, Student Member, IEEE \\ CEGELY, CNRS UMR 5005, INSA-Lyon \\ Building L. De Vinci, 20 avenue A. Einstein, F-69621 Villeurbanne Cedex, France
}

Email: \{xuefang.shi, bruno.allard, dominique.tournier, jean-marie.retif, florent.morel\}@insa-lyon.fr

\begin{abstract}
Analogue control of monolithic DC/DC converters is coming to a limit due to high switching frequency and a request for large regulation bandwidth. Digital control is now experimented for low-power low-voltage switch-mode power supply. Digital implementation of analogue solutions does not prove real performances. Other digital controllers have been experimented but applied to discrete converters. This paper compares a classical digital controller to a possible alternative strategy. Sensitivity functions are used to compare controller performances. RST algorithm determined by robust pole assignment shows better performances.
\end{abstract}

\section{INTRODUCTION}

For many years now, there is a trend to embed power management unit inside portable devices like cellphone, personal digital assistant or MP3-player. Most portable devices use a battery ranging voltage between $5.5 \mathrm{~V}$ when in charge, $3.3 \mathrm{~V}$ during discharge lifetime and down to $2.7 \mathrm{~V}$ when empty. Devices embed various functions supplied from various voltages. Processors require $1.8 \mathrm{~V}$ down to $1.2 \mathrm{~V}$ while backlight led system require $20 \mathrm{~V}$ at least. Non isolated DC/DC converters are considered in place of low-drop out regulators for the sake of efficiency. This paper will now address only stepdown conversion and associated buck architecture or stepdown switch-mode power supply (SMPS).

A full-analogue synchronous buck converter is pictured in Fig. 1. Except the passive L-C output filter, all blocks are integrated monolithically using CMOS standard technology [1], [2]. A 2-poles/2-zeros compensator is implemented to achieve a maximal regulation bandwidth, maximal transient performance and maximal accuracy. The switching frequency must be kept unchanged for EMC filtering purpose.

[3] details a $10 \mathrm{~mW}$ SMPS with $100 \mathrm{MHz}$ switching frequency and $91 \%$ peak efficiency, but poor transient performances. A $100 \mathrm{MHz}$ SMPS, $80 \%$ peak efficiency, $20 \mathrm{MHz}$ regulation bandwidth is presented in [4]. Moreover the design is compatible with standard CMOS process. Whatever analogue control presents some limitations. First of all, the design of the compensator is not automated and the design engineer needs to take care of a trade-off between performances, accuracy and stability [5]. When R-C constant have been set, manufacturing introduces deviation with respect to design values and calibrations are required. So a lot of efforts are put on alternative approaches as digital control.

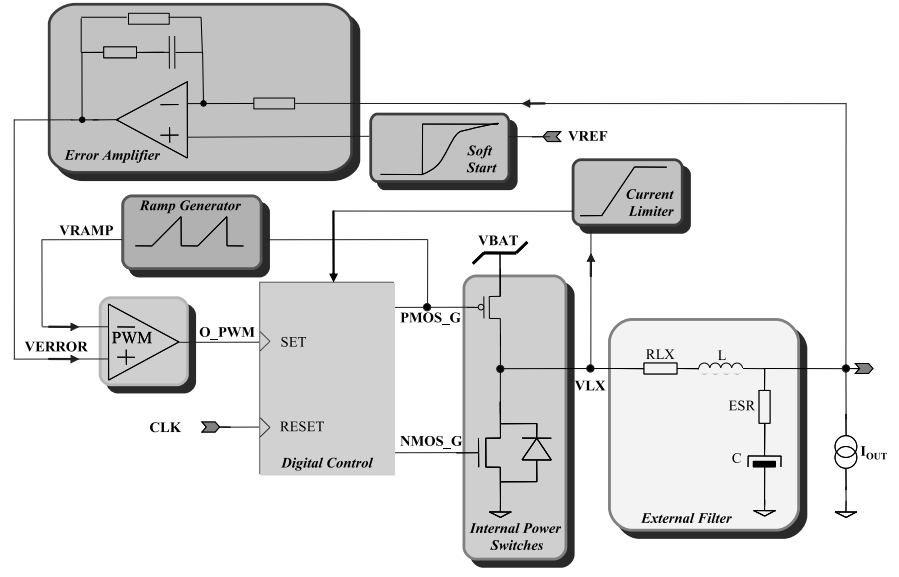

Fig. 1. Schematic synchronous step-down SMPS

Digital control is not new in the field of Power Electronics. It is often associated with DSP or other processorlike implementation [6]-[9]. Generally the digital control system presents sufficient resources to accommodate the modest switching frequency of the converter, in the $\mathrm{kHz}$ range. In embedded applications, switching frequencies in the $\mathrm{MHz}$ and plus range are necessary in order to reduce the size of passive components [10].

Basically most published papers consider a discrete-time equivalent to the analogue compensator [11], [12]. Most efforts are put on a suitable integration of A-to-D converters and the PWM function that is then called a digital PWM (DPWM). Other papers introduce a digital controller based on lookup tables [13]. Complex algorithms have been published but applications concern large-power converters where the energy consumption of the digital controller is nearly negligible with respect to the converter power losses [14]. It is not the case in a low-power sub-1V monolithic SMPS.

Digital-PWM introduces a trade-off between the operating clock frequency and the accuracy. If a ratio of 8-to- 1 is selected with respect to the SMPS switching frequency, then a $10 \mathrm{MHz}$ converter requires a DPWM with a $80 \mathrm{MHz}$ bandwith, and only 255 duty ratio values are acceptable. This kind of DPWM is implemented through a cascade of CMOS inverters where each output is connected to a multiplexer. The DPWM delays an input edge and the MOSFET transistor gate signal is 


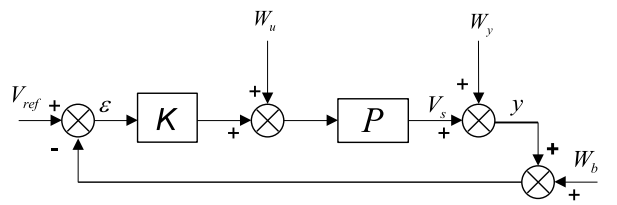

Fig. 2. Block diagram of the SMPS

obtained by logic combination of the initial edge signal with the multiplexer output [12]. The limited number of duty ratio values leads to limit-cycle behaviour of the converter and the accuracy of the output voltage may not be controlled. The Ato-D converter can be replaced by a multi-level comparator and associated to a finite state-machine controller [15]. A $50 \mathrm{MHz}$ digital system is necessary to control a $100 \mathrm{kHz}$ converter with $200 \mathrm{~Hz}$ of regulation bandwidth.

Literature shows that DPWM-less architectures are experimented. Look-up table architecture is then an alternative worth the effort since the power consumption may be limited and at least controlled. Standard CMOS technologies offer high operating frequencies for logic blocks. It is then compatible with $100 \mathrm{MHz}$ converters.

This paper investigates a robust RST control in comparison with a classical PID control. RST is an efficient strategy of digital control that can be applied to monolithic step-down SMPS. Section II introduces so-called sensitivity functions as a quantification mean to compare strategies. Section III introduces a digital PID controller. Section IV introduces a socalled RST digital controller. Simulation results are detailed in Section V.

\section{Sensitivity Functions}

In order to quantify system dynamics, robustness and noise rejection properties of tested controllers, sensitivity functions are introduced.

Fig. 2 represents the z-domain model of SMPS $(P(z))$ and its controller $(K(z))$ when adding control noise $W_{u}$, output noise $W_{y}$ and measurement noise $W_{b}$.

From Fig. 2, it comes the following relation that leads to the sensitivity functions.

$$
\begin{aligned}
y & =\Gamma \cdot V_{\text {ref }}+S_{y y} \cdot W_{y}+S_{y b} \cdot W_{b}+S_{y u} \cdot W_{u} \\
\Gamma & =\frac{K P}{1+K P}=\frac{L_{y y}}{1+L_{y y}} \\
S_{y y} & =\frac{1}{1+K P}=\frac{1}{1+L_{y y}} \\
S_{y b} & =\frac{-K P}{1+K P}=\frac{-L_{y y}}{1+L_{y y}} \\
S_{y u} & =\frac{P}{1+K P}=\frac{P}{1+L_{y y}}
\end{aligned}
$$

where $\Gamma$ is the closed loop transfer function, $S_{y y}, S_{y b}$ and $S_{y u}$ are respectively the output-to-output, measure to output and control-to-output sensibility functions.

Constraints or disturbance rejections are naturally expressed in terms of frequency sensitivity shapes. For a given controller,

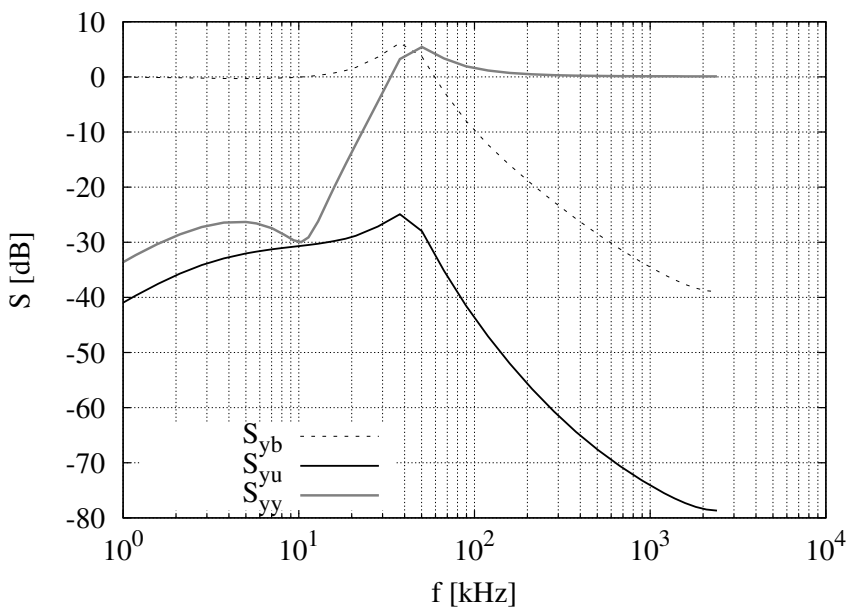

Fig. 3. Sensitivity Functions Example

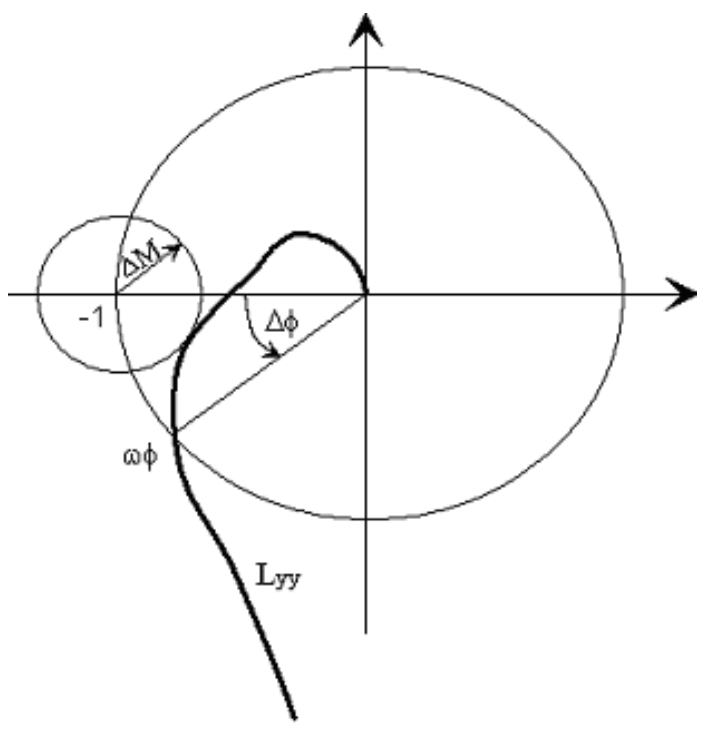

Fig. 4. Typical Nyquist plot of $L_{y y}$

the sensitivity functions allow to evaluate the controller behavior in relation to the desired attenuation constraints.

Fig. 3 is an example of the gain plot of sensibility functions. The gradient of $S_{y y}$ at low frequency determines the dynamic behavior of the system. The bandwidth of $S_{y b}$ defines the influence of noise on the output voltage and the closed loop bandwidth since it has the same transfer function as $\Gamma$ expect the sign. The gain of $S_{y u}$ verifies the rejection of control perturbations such as the PWM-related noises.

In addition, from the maximum value of $S_{y y}$, the margin module can be determined. The module margin $\Delta \mathrm{M}$ is defined as the minimum distance of $L_{y y}$ to the critical locus -1 in the Nyquist plan (Fig. 4). The module margin and delay margin quantify the robustness of the modeling uncertainties. The delay margin $\Delta \tau$ is deduced from the phase margin $\Delta \Phi$ by $\Delta \tau=\frac{\Delta \Phi}{\omega_{\Phi}}$.

In order to ensure robustness, the module margin $\Delta \mathrm{M}$ is 
kept higher than 0.5 and the delay margin must be higher than sampling period.

\section{PID CONTROL}

The PID controller is presented for comparison purpose with the RST controller. A digitally controlled buck converter operating in continuous conduction mode (CCM) can be regarded as a second order discrete-time system [16]:

$$
P(z)=\frac{b_{1} z+b_{2}}{z^{2}+a_{1} z+a_{2}}
$$

A discrete-time PID controller can be written as:

$$
K_{P I D}(z)=\frac{r_{0} z^{2}+r_{1} z+r_{2}}{(z-1)\left(z+s_{1}\right)}
$$

where $r_{0}, r_{1}, r_{2}, s_{1}$ are the controller parameters to be determined.

By cancelling the poles of $P(z)$ with the zeros of $K(z)$, the closed-loop reference to output voltage transfer function is:

$$
\begin{aligned}
\Gamma & =\frac{K P}{1+K P} \\
& =\frac{r_{0} b_{1} z+r_{0} b_{2}}{z^{2}+\left(s_{1}-1+r_{0} b_{1}\right) z+\left(r_{0} b_{2}-s_{1}\right)} \\
& =\frac{r_{0} b_{1} z+r_{0} b_{2}}{z^{2}+p_{1} z+p_{2}}
\end{aligned}
$$

$p_{1}$ and $p_{2}$ are defined by the desired closed-loop dynamics which corresponds to a second order dynamics with a pulsation $\omega_{c l}$ and a damping ratio $\xi_{c l}$. The conditions for the cancellation of the poles of $P(z)$ by the zeros of $K(z)$ are:

$$
\begin{aligned}
& r_{0}=\frac{1+p_{1}+p_{2}}{b_{1}+b_{2}} \\
& r_{1}=a_{1} r_{0} \\
& r_{2}=a_{2} r_{0} \\
& s_{1}=r_{0} b_{2}-p_{2}
\end{aligned}
$$

In order to have a large enough closed-loop bandwidth, the desired closed-loop dynamic is set to about 15 times the openloop dynamic and a damping ratio of 0.7 .

For the circuit elements with $\mathrm{L}=10 \mu \mathrm{H}, \mathrm{C}=22 \mu \mathrm{F}$, $\mathrm{R}=3 \Omega, \mathrm{V}_{B A T}=3.3 \mathrm{~V}$, the controller parameters are: $r_{0}=431.64, r_{1}=-860.28, r_{2}=428.73$ and $s_{1}=-0.83$. The corresponding sensitivity functions are presented in Fig. 5.

For an output disturbance with a pulsation of $\omega_{1}$ (LC filter resonance), the gain of $S_{y y}$ on $\omega_{1}$ gives the information on the disturbance rejection. In the study case, $\omega_{1}=10.568 \mathrm{kHz}$. It can be seen that $S_{y y}\left(\omega_{1}\right)$ is about $-25 \mathrm{~dB}$.

Concerning the stability robustness, the module, phase and delay margins are: $\Delta \mathrm{M}=0.88, \Delta \varphi=66.9^{\circ}, \Delta \tau=6.6 \mathrm{~T}_{e}$.

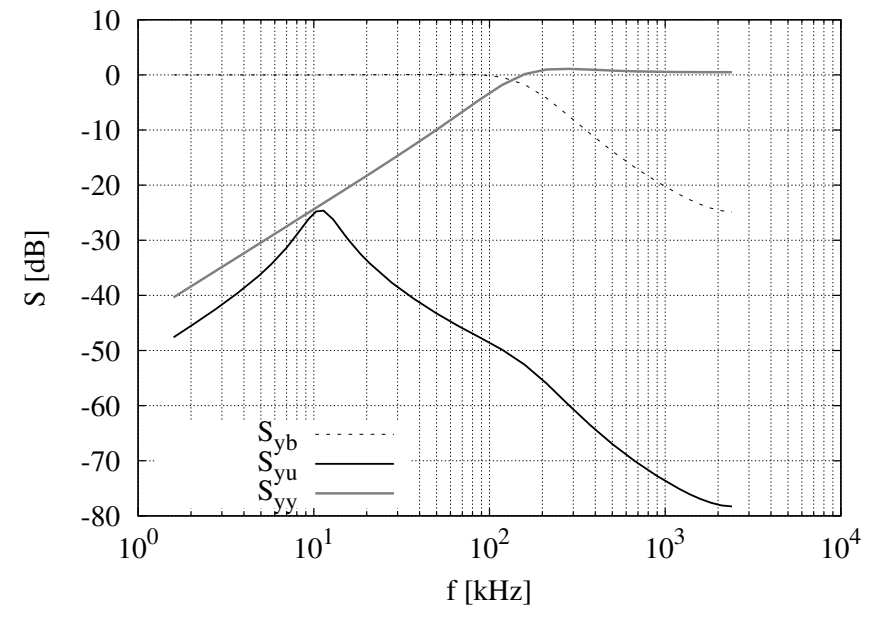

Fig. 5. Sensitivity functions for PID controlled system

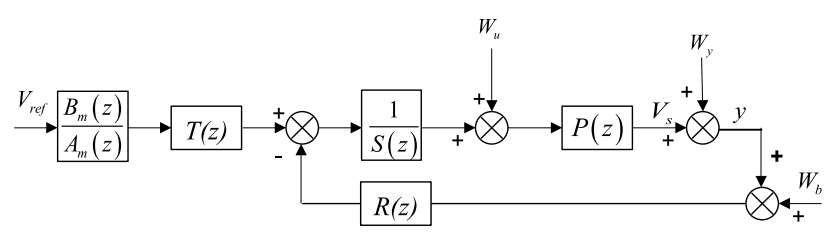

Fig. 6. RST control structure

\section{ROBUST RST CONTROL}

RST control realizes a relevant approach for linear Single Input Single Output (SISO) systems [17]. The structure of a RST control is presented in Fig. 6.

The sensitivity functions can be expressed as:

$$
\begin{aligned}
S_{y y} & =\frac{A S}{A S+B R} \\
S_{y b} & =\frac{-B R}{A S+B R} \\
S_{y u} & =\frac{B S}{A S+B R}
\end{aligned}
$$

From these expressions, it can be noted that the three sensitivity functions have the same denominator $D=A S+$ $B R$ which determines the dynamics of disturbance rejections. The knowledge of acceptable disturbances leads to design the RST controller in terms of poles and zeros assignments. For example, to insure the output accuracy, a pole for $z=1$ in $S(z)$ is necessary for static error elimination.

For an output disturbance with the pulsation of $\omega_{1}$, the lower gain of $S_{y y}$ at $\omega_{1}$, better the attenuation of the output disturbance rejection. However, the diminution of $S_{y y}$ at $\omega_{1}$ can induce an augmentation of the maximum value of $S_{y y}$ which is inversely proportional to $\Delta \mathrm{M}$. The diminution of $\Delta \mathrm{M}$ reduces the controller robustness.

How to determine a controller which makes a compromise between the robustness and a good rejection of disturbances? This can be translated as an optimization problem by defining 


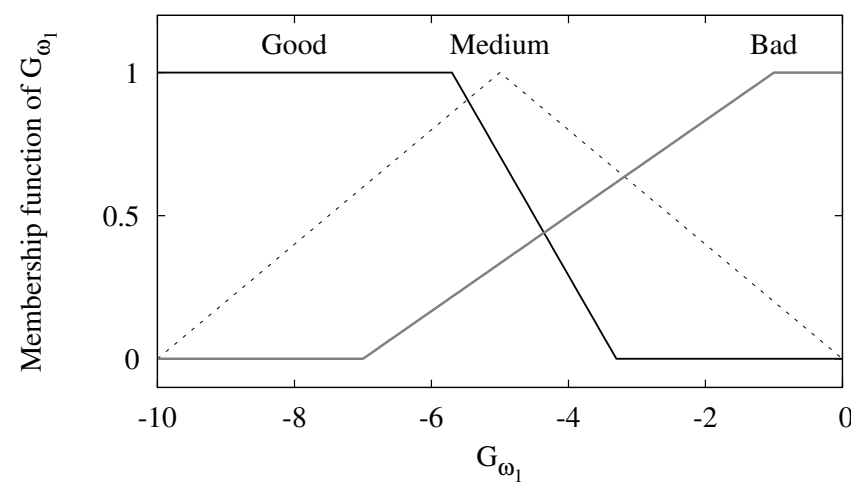

Fig. 7. Input membership function for $G_{\omega_{1}}$

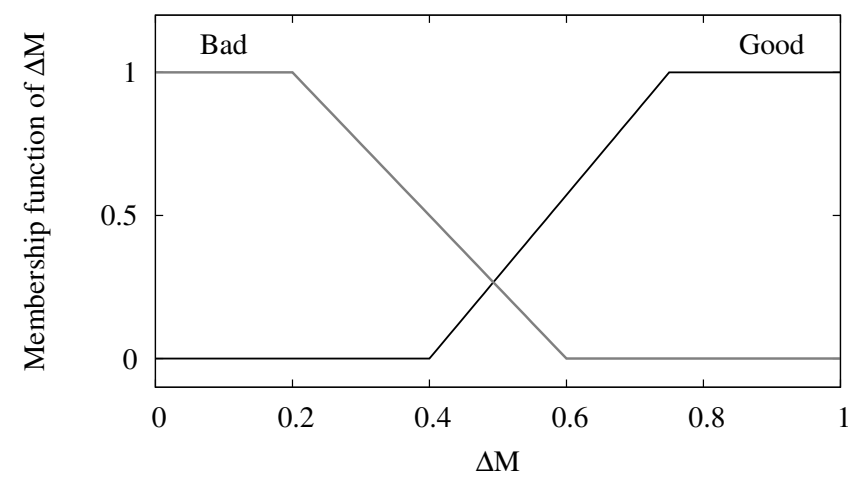

Fig. 8. Input membership function for $\Delta \mathrm{M}$

a cost function which qualifies the controller robustness and noise rejection properties.

Fuzzy logic is more suitable to qualify the robustness of a controller and noise rejection properties [18]. Indeed the frontier between a good controller and a bad controller is not strict. For example, if one considers the module margin is correct if it is higher than 0.5 , it is clear that a controller will not be qualified as a bad robustness to good by crossing this value. With membership functions of fuzzy logic, the controller quality can be evaluated continually from bad to good across medium.

An example of membership functions qualified the gain at $\omega_{1}\left(G_{\omega_{1}}\right)$ and the module margin $(\Delta \mathrm{M})$ is shown in Fig. 7 and Fig. 8 .

$G_{\omega_{1}}$ is normed on $\left\{\begin{array}{lll}-10 & 0\end{array}\right.$ and $\Delta \mathrm{M}$ uses its natural scale.

The output membership function is given in Fig. 9.

The stability robustness can be expressed by the fuzzy rules defined in Tab. I.

TABLE I

FUZZY RULES USED

\begin{tabular}{|c|c|c|c|}
\hline$\Delta \mathrm{M} \quad G_{\omega_{1}}$ & Bad & Medium & Good \\
\hline$\overline{\text { Bad }}$ & $\overline{\mathrm{Bad}}$ & $\overline{\mathrm{Bad}}$ & Medium \\
\hline Good & $\mathrm{Bad}$ & Medium & Good \\
\hline
\end{tabular}

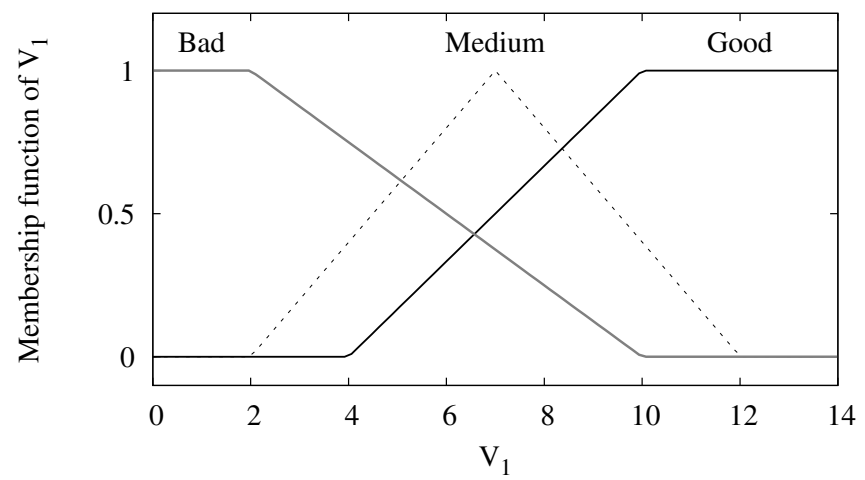

Fig. 9. Output membership functions

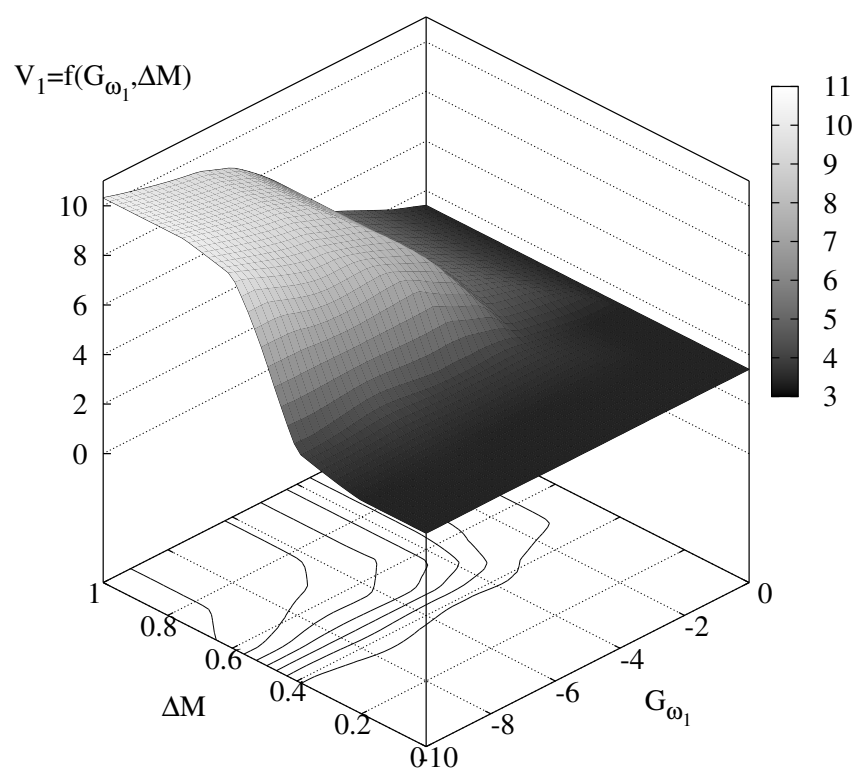

Fig. 10. Fuzzy function $V_{1}$

After "disfuzzyfication", the robustness analyse is quantified by the function $V_{1}=\mathrm{f}\left(G_{\omega_{1}}, \Delta \mathrm{M}\right)$ which corresponds to a surface as presented in Fig. 10. It can be seen that for $\Delta \mathrm{M}>0.5$, the lower the values of $G_{\omega_{1}}$, the better the output function $V_{1}$.

On the same way, other membership functions and fuzzy rules in relation with delay margin and with other sensitivity functions can be defined to quantify constraints of robustness and satisfies the requirements of disturbance rejection performances. The cost function to maximize is a balanced sum of all fuzzy functions. The optimization problem is realized by a stochastic genetic algorithm [19]. The computation time can be important but it is done off line, so that is not a limitation for real time implementation.

For our example, the denominator of the sensitivity function 


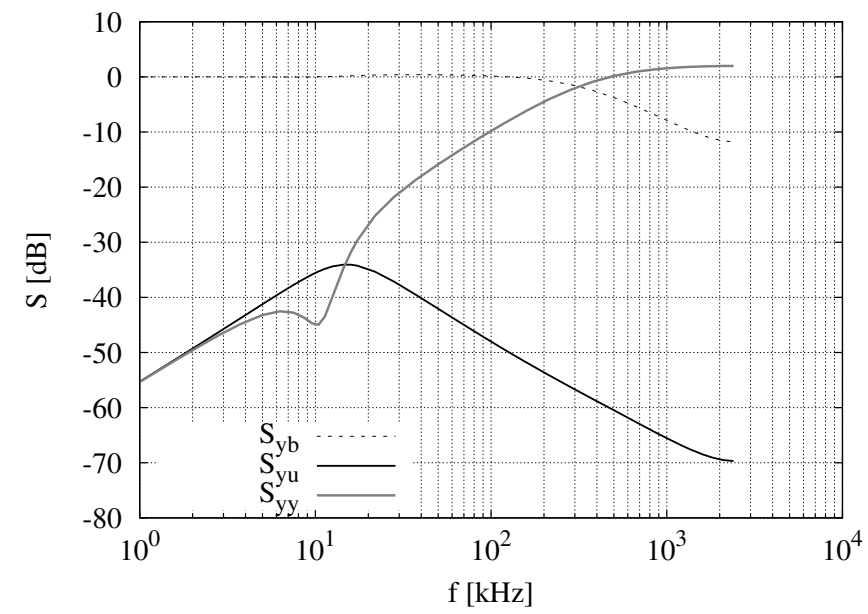

Fig. 11. Sensitivity functions for RST controller

$S_{y y}$ is defined as follows to determine a RST controller which presents a good robustness and a good attenuation of the influence of the output disturbance at $\omega_{1}=10.568 \mathrm{kHz}$.

$$
D(z)=\left(1-c_{0} z^{-1}\right)\left(1+c_{1} z^{-1}+c_{2} z^{-2}\right)
$$

where $c_{0}, c_{1}$ and $c_{2}$ constitute three degrees of freedom for the optimization problem. For each combination of $c_{0}, c_{1}$ and $c_{2}$, the fuzzy logic quantifiers evaluate the cost function. The genetic algorithm then finds the optimal solution for $c_{0}, c_{1}$ and $c_{2}$ over twenty generations with about thirty individuals. The corresponding optimal polynoms $R(z), S(z)$ and $T(z)$ are calculated resolving the Bezout equation [17].

$$
\begin{aligned}
& R(z)=669.2 z^{2}-1321.9 z+653 \\
& S(z)=z^{2}-1.6815 z+0.6815 \\
& T(z)=1617.9 z^{3}-4176.2 z^{2}+3515.6 z-957.1
\end{aligned}
$$

The corresponding sensitivity functions are given in Fig. 11.

The gain of $S_{y y}$ on $\omega_{1}$ is $-39 \mathrm{~dB}$ against $-25 \mathrm{~dB}$ for PID controller. The good robustness is keep with $\Delta \mathrm{M}=0.79$, $\Delta \varphi=75.3^{\circ}$ and $\Delta \tau=3.18 \mathrm{~T}_{e}$.

\section{Simulation Results}

The simulations are done in Simulink 12. The modeling of the buck SMPS is with a hybrid model [5]. The PID controller and RST controller are modeled with standard elements of the Simulink library. Analog-to-digital converter model takes into account quantization, delay and saturation effects. The DPWM model has an 8-bit resolution [12].

After a reference tracking, a step change of load from $0 \mathrm{~A}$ to $0.6 \mathrm{~A}$ is applied at $400 \mu \mathrm{s}$. The output voltage is compared in Fig. 13 and Fig. 14. The load transient responses of RST controller is superior to PID controller since it results in shorter rise time and produces smaller undershoot.

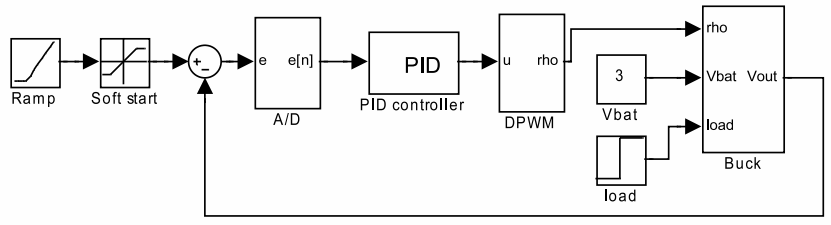

Fig. 12. Simulink model of PID digital controller for a buck converter

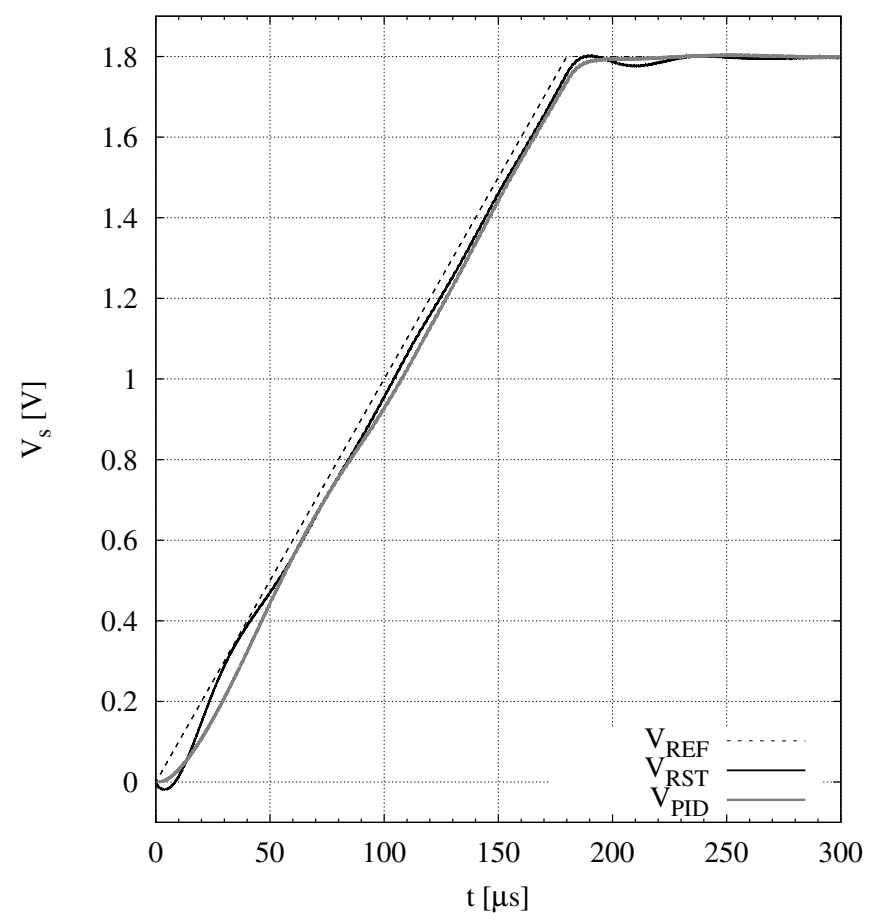

Fig. 13. Comparison of output voltage responses during reference tracking

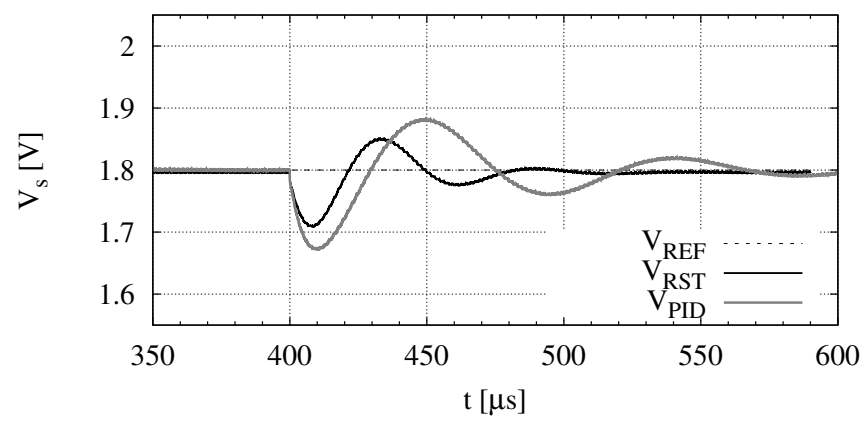

Fig. 14. Comparison of output voltage responses to load perturbation 


\section{CONCLUSION}

This paper has detailed the design of a now classical PID digital controller and one alternative as the RST strategy. RST offers better performances. Simulation results have concerned a $600 \mathrm{kHz}$ switching frequency converter, but results hold for higher switching frequencies. Superior performances are noted for a $10 \mathrm{MHz}$ buck converter. Moreover RST implementation can be achieved using one A-to-D converter and standard CMOS logic. Experimental study are currently performed using an Actel ProAsic3 FPGA board plus an additional analog I/O board. It is possible to estimate the impact of monolithic integration from experimental results as FPGA design can be translated at no cost into an ASIC version. PID like RST controller requires a fast A-to-D converter. It will probably be the angrier energy consumer in the digital control. Other alternative digital strategies are possible like hybrid approaches or direct voltage control.

\section{REFERENCES}

[1] V. Kursun, S. Narendra, V. K. De, and E. Friedman, "High input voltage step-down dc/dc converters for integration in a low voltage $\mathrm{cmos}$ process," Proc. of IEEE ISCAS, 2004.

[2] H. Deng, X. Duan, Y. Ma, A. Huang, and D. Chen, "Monolithically integrated boost converter based on 0.5 um cmos process," IEEE Transactions on Power electronics, vol. 20, no. 3, pp. 628-638, 2005.

[3] T. Fuse, M. Ohta, M. Tokumasu, H. Fujii, S. Kawanaka, and A. Kameyama, "A $0.5 \mathrm{v}$ power-supply scheme for low-power system lsis using multi-vth soi-cmos technology," IEEE Journal of Solid-State Circuits, vol. 38, no. 2, pp. 303-311, 2003.

[4] V. Pinon, B. Allard, and C. Garnier, "High-frequency monolithic $\mathrm{dc} / \mathrm{dc}$ converter for system-on-chip power management high-frequency monolithic $\mathrm{dc} / \mathrm{dc}$ converter for system-on-chip power management," Proceedings of IEEE ISPSD, Naples, June, 2006, to be published.

[5] B. Allard, S. Trochut, X. Lin-Shi, and J. M. Rétif, "Control design for integrated switch-mode power supplies : a new challenge ?" Proc. of the IEEE Power Electronics Specialist's Conference, 2004, cdrom.
[6] T. Martin and S. Ang, "Digital control for switching converters," Proc of IEEE ISIE, vol. 2, pp. 480-484, 1995.

[7] H. Matsuo, Y. Mimura, Y. Nakao, F. Kurokawa, and M. Sasaki, "Novel digital controller for the pwm and/or pfm controlled dwitching $\mathrm{dc} / \mathrm{dc}$ converters," Proc of IEEE INTELEC, vol. 2, pp. 65-68, 2001.

[8] J. L. G. Hung and R. Nelms, "Pid controller modifications to improve steady-state performance of digital controllers for buck and boost converters," Proc of IEEE APEC, vol. 1, pp. 381-388, 2002.

[9] D. Maksimovic, R. Zane, and R. Erickson, "Impact of digital control in power electronics," Proc of IEEE ISPSD, pp. 13-22, 2004.

[10] S. Musunuri, P. Chapman, J. Zou, and C. Liu, "Design issues for monolithic dc-dc converters," IEEE Transactions on Power electronics, vol. 20 , no. 3, pp. 639-649, 2005.

[11] G. Wei and M. Horowitz, "A fully digital, energy-efficient, adaptative power-supply regulator," IEEE Journal of Solid-State Circuits, vol. 34, no. 4, pp. 520-528, 1999.

[12] B. J. Patella, A. Prodic, A. Zirger, and D. Maksimovic, "High-frequency digital pwm controller ic for dc-dc converters," IEEE Transactions on Power electronics, vol. 18, no. 1, pp. 438-446, 2003.

[13] A. Peterchev and S. Sanders, "Digital loss-minimizing multi-mode synchronous buck converter control," Proc. of the IEEE Power Electronics Specialist's Conference, no. 3694-3699, 2004.

[14] S. Saggini, M. Ghiono, and A. Geraci, "An innovative digital control architecture for low-voltage, high-current dc-dc converters with tight voltage regulation," IEEE Transactions on Power electronics, vol. 19, no. 1, pp. 210-217, 2004.

[15] M. Islam, D. Allee, S. Konasani, and A. Rodriguez, "A low-cost digital controller for a switching dc converter with improved voltage regulation," IEEE Power Electronics Letters, vol. 2, no. 4, pp. 121-124, 2004.

[16] R. W. Erickson and D. Maksimovic, Fundamentals of Power Electronics. Kluwer, 2001, 2nd edition.

[17] I. Landau, Identification et commande des systémes. Hermes, 1993, 2nd edition.

[18] C.C.Lee, "Fuzzy logic in control systems: Fuzzy logic controller-part i," IEEE Trans. On system, Man and Cybernetics, vol. 20, no. 2, pp. 404-408, 1990.

[19] D.Beasley and al., "An overview of genetic algorithms: Part 1, fundamentals," University Computing, vol. 15, no. 2, pp. 58-69, 1993. 